\title{
Correction to: Investigating psychometric properties of the Thai version of the Zarit Burden Interview using rasch model and confirmatory factor analysis
}

Kanokporn Pinyopornpanish ${ }^{1}$, Manee Pinyopornpanish ${ }^{2}$, Nahathai Wongpakaran²*0, Tinakon Wongpakaran², Atiwat Soontornpun ${ }^{3}$ and Pimolpun Kuntawong ${ }^{2}$

\section{Correction to: BMC Res Notes (2020) 13:120} https://doi.org/10.1186/s13104-020-04967-w

Following the publication of the original article [1], the authors unfortunately became aware of the following errors in Table 3:

\#21 in ZBI-22 should be corrected from 'should do more' to 'could do a better job'.

\#12 in ZBI-12 should be corrected from 'should do more' to 'could do a better job'.

The corrected Table 3 is shown below:

The original article can be found online at https://doi.org/10.1186/s1310 4-020-04967-w.

*Correspondence: nahathai.wongpakaran@cmu.ac.th

2 Department of Psychiatry, Faculty of Medicine, Chiang Mai University,

110 Intawaroros Rd. Tambon Sriphoom, Amphur Mueng, Chiang

Mai 50200, Thailand

Full list of author information is available at the end of the article

C The Author(s) 2020. This article is licensed under a Creative Commons Attribution 4.0 International License, which permits use, sharing, adaptation, distribution and reproduction in any medium or format, as long as you give appropriate credit to the original author(s) and the source, provide a link to the Creative Commons licence, and indicate if changes were made. The images or other third party material in this article are included in the article's Creative Commons licence, unless indicated otherwise in a credit line to the material. If material is not included in the article's Creative Commons licence and your intended use is not permitted by statutory regulation or exceeds the permitted use, you will need to obtain permission directly from the copyright holder. To view a copy of this licence, visit http://creativecommons.org/licenses/by/4.0/. The Creative Commons Public Domain Dedication waiver (http://creativecommons.org/publicdomain/zero/1.0/) applies to the data made available in this article, unless otherwise stated in a credit line to the data. 
Table 3 Rasch analysis results of the ZBI

\begin{tabular}{|c|c|c|c|c|c|c|c|c|c|c|c|}
\hline \multicolumn{6}{|l|}{ ZBI-22 } & \multicolumn{6}{|c|}{ ZBI-12 } \\
\hline \multirow{2}{*}{$\begin{array}{l}\text { No. } \\
\text { 1. Asks for more } \\
\text { help than needs }\end{array}$} & \multirow{2}{*}{$\begin{array}{l}\text { Measure } \\
0.44\end{array}$} & \multicolumn{2}{|c|}{ INFIT MNSQ } & \multirow{2}{*}{$\begin{array}{l}\text { OUTFIT MNSQ } \\
1.453\end{array}$} & \multirow{2}{*}{$\begin{array}{l}\text { Disordered } \\
\text { category } \\
\text { or threshold }\end{array}$} & \multirow[t]{2}{*}{ No. } & \multicolumn{2}{|c|}{ Measure } & \multirow[t]{2}{*}{ INFIT MNSQ } & \multirow[t]{2}{*}{ OUTFIT MNSQ } & \multirow{2}{*}{$\begin{array}{l}\text { Disordered } \\
\text { category } \\
\text { or threshold }\end{array}$} \\
\hline & & 1.321 & & & & & & & & & \\
\hline $\begin{array}{l}\text { 2. Not have enough } \\
\text { time }\end{array}$ & 0.04 & 0.912 & & 0.859 & No & 1 & -0.1 & & 0.903 & 0.897 & No \\
\hline 3. Feel stressed & -0.03 & 0.697 & & 0.614 & Yes & 2 & -0.6 & & 0.905 & 0.865 & No \\
\hline 4. Feel embarrassed & 0.14 & 1.120 & & 1.099 & Yes & & & & & & \\
\hline 5. Feel angry & 0.13 & 0.891 & & 0.932 & No & 3 & 0.40 & & 0.978 & 1.014 & No \\
\hline $\begin{array}{l}\text { 6. Negative rela- } \\
\text { tionships }\end{array}$ & 0.37 & 0.941 & & 0.956 & Yes & 4 & 0.67 & & 1.129 & 0.855 & Yes \\
\hline $\begin{array}{l}\text { 7. Afraid about the } \\
\text { future }\end{array}$ & -0.64 & 1.082 & & 1.134 & No & & & & & & \\
\hline $\begin{array}{l}\text { 8. Dependent on } \\
\text { you }\end{array}$ & -0.92 & 1.258 & & 1.334 & Yes & & & & & & \\
\hline 9. Feel strained & 0.23 & 0.760 & & 0.655 & No & 5 & 0.33 & & 0.818 & 0.781 & Yes \\
\hline $\begin{array}{l}\text { 10. Health } \\
\text { decreased }\end{array}$ & 0.31 & 0.646 & & 0.795 & No & 6 & 0.40 & & 0.806 & 1.083 & No \\
\hline 11. Lack of privacy & -0.04 & 0.616 & & 0.730 & No & 7 & 0.11 & & 0.893 & 1.062 & No \\
\hline 12. Lack of social life & 0.19 & 0.675 & & 0.639 & No & 8 & 0.32 & & 0.701 & 0.682 & No \\
\hline $\begin{array}{l}\text { 13. Feel uncomfort- } \\
\text { able }\end{array}$ & 0.38 & 0.943 & & 1.967 & No & & & & & & \\
\hline $\begin{array}{l}\text { 14. Expecting to be } \\
\text { cared for }\end{array}$ & -0.58 & 1.512 & & 1.612 & Yes & & & & & & \\
\hline 15. Lack of money & 0.12 & 1.355 & & 1.399 & No & & & & & & \\
\hline $\begin{array}{l}\text { 16. Unable to care } \\
\text { much longer }\end{array}$ & 0.72 & 0.789 & & 0.777 & Yes & & & & & & \\
\hline $\begin{array}{l}\text { 17. Lost control } \\
\text { of life }\end{array}$ & 0.60 & 0.844 & & 0.737 & Yes & 9 & 0.35 & & 0.918 & 0.796 & Yes \\
\hline 18. Leave the care & 0.40 & 1.423 & & 1.358 & Yes & & & & & & \\
\hline $\begin{array}{l}\text { 19. Uncertain about } \\
\text { what to do }\end{array}$ & 0.14 & 0.795 & & 0.704 & Yes & 10 & 0.40 & & 1.035 & 1.029 & Yes \\
\hline 20. Should do more & -0.71 & 1.264 & & 1.256 & No & 11 & -1.0 & & 1.406 & 1.339 & No \\
\hline $\begin{array}{l}\text { 21. Could do a bet- } \\
\text { ter job caring }\end{array}$ & -0.80 & 1.383 & & 1.367 & No & 12 & -1.0 & & 1.449 & 1.317 & No \\
\hline $\begin{array}{l}\text { 22. Overall feeling } \\
\text { of burden }\end{array}$ & -0.50 & 0.912 & & 0.973 & Yes & & & & & & \\
\hline \multicolumn{3}{|l|}{ PCA } & \multicolumn{3}{|c|}{ ZBI-22 } & \multicolumn{4}{|c|}{ ZBI-12 } & & \\
\hline \multicolumn{3}{|c|}{ \%Variance explained by measures } & \multicolumn{3}{|c|}{$46.0 \%$} & \multicolumn{4}{|c|}{$48.7 \%$} & & \\
\hline \multicolumn{3}{|c|}{ Unexplained variance in first contrast } & \multicolumn{3}{|c|}{$2.52(6.2 \%)$} & \multicolumn{4}{|c|}{$3.03(12.6 \%)$} & & \\
\hline \multicolumn{3}{|c|}{ Disattenuated correlation } & \multicolumn{3}{|c|}{$0.896-1.000$} & \multicolumn{4}{|c|}{$0.357-1.000$} & & \\
\hline \multicolumn{3}{|c|}{$\begin{array}{l}\text { Pair of item that standardized residual } \\
\text { correlation }>0.2\end{array}$} & \multicolumn{5}{|c|}{$\begin{array}{l}\text { \#20(should do more)—\#21(could do a better job) \#11(lack } \\
\text { of privacy)—\#12(lack of social life) \#1 (asks for more help } \\
\text { than needs)—\#18(leave the care) \#15(lack of money)—- } \\
\text { \#16(unable to care much longer) \#5(feel angry)—\#9(feel } \\
\text { strained) }\end{array}$} & \multicolumn{4}{|c|}{$\begin{array}{l}\# 11 \text { (should do more)—\#12(could do a better job) \#3(feel } \\
\text { angry)—\#5(feel strained) \#8(lack of privacy)—\#9(lack } \\
\text { of social life) }\end{array}$} \\
\hline \multicolumn{3}{|c|}{ Item separation/reliability } & $2.57 / C$ & & & & & $2.03 /$ & 0.80 & & \\
\hline Person separation/rel & liability & & $4.08 / C$ & & & & & $3.97 /$ & 0.94 & & \\
\hline
\end{tabular}

ZBI, Zarit Burden Interview; PCA, Principal component analysis; INFIT, inlier-pattern-sensitive fit statistic; OUTFIT, outlier-sensitive fit statistic; MNSQ, mean-square; \#, item number 


\section{Author details}

${ }^{1}$ Department of Family Medicine, Faculty of Medicine, Chiang Mai University, Chiang Mai, Thailand. ${ }^{2}$ Department of Psychiatry, Faculty of Medicine, Chiang Mai University, 110 Intawaroros Rd. Tambon Sriphoom, Amphur Mueng, Chiang Mai 50200, Thailand. ${ }^{3}$ Division of Neurology, Department of Internal Medicine, Faculty of Medicine, Chiang Mai University, Chiang Mai, Thailand.

Published online: 20 April 2020

\section{Reference}

1. Pinyopornpanish K, Pinyopornpanish M, Wongpakaran N, Wongpakaran T, Soontornpun A, Kuntawong P. Investigating psychometric properties of the Thai version of the Zarit Burden Interview using rasch model and confirmatory factor analysis. BMC Res Notes. 2020;13:120. https://doi. org/10.1186/s13104-020-04967-w.

\section{Publisher's Note}

Springer Nature remains neutral with regard to jurisdictional claims in published maps and institutional affiliations.
Ready to submit your research? Choose BMC and benefit from:

- fast, convenient online submission

- thorough peer review by experienced researchers in your field

- rapid publication on acceptance

- support for research data, including large and complex data types

- gold Open Access which fosters wider collaboration and increased citations

- maximum visibility for your research: over 100M website views per year

At BMC, research is always in progress.

Learn more biomedcentral.com/submissions 